\title{
Rilevamento geomagnetico degli apparati vulcanici Vicano e Sabazio
}

\author{
(Geomagnetic Survey of Vicano and Sabazio volcanic apparatus)
}

\author{
F. Molina $(*)-$ A. Sonaglia $(* *)$
}

Ricevuto il 19 Luglio 1969

\begin{abstract}
Riassunto. - Un rilevamento geomagnetico per la componente verticale dol campo magnetico terrestre è stato effettuato nella regione interessata dagli apparati vulcanici Vicano e Sabazio, per uno studio più approfondito delle masse magmatiche che hamo alimentato tali apparati.

L'interpretazione dei risultati ha portato alle seguenti conclusioni: a) esiste una discontinuiti fra la massa magnatica che ha alimentato il vulcanismo Vicano e Sabazio e quelle responsabili del vulcanismo relativo all'apparato Vulsino e all'apparato Laziale; b) tale massa magmatica può essere assimilata ad un corpo approssimativamente ellissoidale, avente il centro fra i $7 \mathrm{~km}$ e i $10 \mathrm{~km}$ di profondità e la superficie superiore molto vicina a quella del suolo, e dimensioni laterali dell'ordine dei $12.15 \mathrm{~km}$, che presenta delle protuberanze verso l'alto corrispondenti al cratere del lago di Vico e alla zona a nord-ovest della conca del lago di Bracciano; $c$ ) esiste una apofisi laterale, collegata alla massa principale, che interessa, fra le altre, le conche crateriche di Polline, Martignano, Baccano e Sacrofano, costituita da una specie di piastra poco spessa estendentesi verso est ad una profonditi dell'ordine di $1-2 \mathrm{~km}$, della larghezza di circa $5 \mathrm{~km}$.
\end{abstract}

SUmmary. - A geomagnetic survey for the vertical component of the earth's field has been made in the region of lakes Vico and Bracciano, i. e., in the areas interested by the Vican and Sabatian voleanic structures, in view of a more detailed study of the magmatic masses that have alimented these structures.

Interpretations of the results have led to following conclusion: a) a discontinnity exists between the magmatic mass that has alimented the Vican

(*) Istituto Nazionale di Geofisica.

(**) Centro di Geologia Tecnica (C.N.R.) - Istituto di Geologia Applicata all'Ingegneria dell'Università di Roma. 
and Sabatian volcanism and the magmatic masses responsible for the vol canism in the area of lake Bolsena and of the Colli Albani: b) this magmatic mass may be assimilated to a rougly ellipsoidal borly with the central point at approximately 7 to $10 \mathrm{~km}$ depth and the upper level very near to surface, and with lateral dimensions of the order of about $12-15 \mathrm{~km}$, presenting upward protuberances that correspond to the crater of the Vico lake and to the area on the northwest of the Bracciano lake basin; $c$ ) there exist a lateral apophysis, connected with the main mass, which interests the craters of Polline, Hartignano, Baccano, and Sacrofano; it consists of a kind of plate of minor thickness, extending eastward at a depth of about $1-2 \mathrm{~km}$. and roughly $5 \mathrm{~km}$ large.

Nel quadro delle ricerche sui vulcani dell'Italia Centrale, che si stamno svolgendo da tempo presso l'Istituto di Geologia Applicata all'Ingegneria dell'Università di Roma, si è prospettata l'utilità di approfondire lo studio sulle caratteristiche di forma, dimensione, e profondità delle masse che hanno alimentato l'attività degli apparati vulcanici Cimino, Vicano, Cerite e Tolfetano.

\section{1. - Cenni geologici.}

All'inizio di questo studio è necessario esporre, sia pur sommariamente, le linee essenziali della geologia della zona.

Il fenomeno vulcanico che ha portato alla caratterizzazione degli apparati Cimino, Vicano, Sabazio, Cerite e Tolfetano ha interessato un basamento sedimentario marino i cui termini più antichi sono rappresentati dalle rocee calcaree e calcareo-dolomitiche mesozoiche del MI. Soratte e MI. Le Fate.

I termini successivi in ordine cronologico sono: la formazione flyscioide affiorante con discreta continuità, ad occidente, lungo una fascia compresa fra Vetralla e S. Severa, quindi il gruppo delle argille, sabbie e conglomerati del pliocene e del pleistocene. Quest'ultima formazione, affiorando con continuita nel tratto orientale ed in maniera sporadica nelle vallate delimitate dai rilievi flyscioidi nel tratto occidentale, circonda la zona vulcanica e costituisce il termine più recente del basamento prevulcanico.

Il fenomeno vulcanico che ha interessato l'area in esame è caratterizzato da due tipi di vulcaniti, l'uno a chimismo acido come gli apparati del M. Cimino, di La Tolfa e del gruppo dei Ceriti, oltre alcuni rilievi presso Manziana (ad ovest di Bracciano), l'altro a chi- 
mismo basico come il centro Vicano ed i numerosi centri dellarea Sabazia. Il vulcanismo basico, predominante nella zona, è stato essenzialmente caratterizzato dai due centri, quello Vicano e quello Sabazio, che differiscono notevolmente fra loro; infatti mentre nel gruppo Vieano si riscontra un unico centro di emissione di lave che ha interealato fasi esplosive con lancio di prodotti piroclastici, nel gruppo Sabazio sono stati accertati numerosi punti di efiusione, che sono probabilmente collegabili a discontinuità del substrato.

Infine interessano la zona numerose "placche» di travertino, come quella molto ampia ad ovest di Viterbo, gli afforamenti a nord-ovest della zona in studio, l'affioramento di Pian Sultano (S. Severa), quelli fra Orte e Gallese e gli altri nei pressi del M. Soratte.

L'analisi dei dati geologici pone dei problemi sui rapporti profondi fra gli apparati Vicano e Sabazio con quello Vulsino ed il Vulcano Laziale, sulla forma, dimensione ed estensione in profonditi della massa o delle masse magmatiche che hamo alimentato i centri di Vico e di Bracciano, nonché i rapporti fra i due apparati stessi.

\section{2. - METODo DI S'TUDIo.}

Per dare un contributo alla soluzione di questi problemi, si è effettuato uno studio geofisico, mediante un rilevamento geomagnetico per la componente verticale $Z$ del campo magnetico terrestre.

La regione studiata interessa 56 tavolette $1 / 25.000$ dell'I.G.AI. Il rilevamento magnetico ì stato eseguito con un magnetometro a torsione GfZ - Askania avente una costante di scala di $2 \tau \gamma / 0$.

Come è noto, questo strumento permette una notevole rapidità di lavoro unita ad una considerevole precisione: dalla ripetizione di numerose (313) stazioni si è dedotto un errore medio per la singola misura di $\pm 3 \gamma$. Nei limiti del possibile, si è cereato di eseguire in tutta la regione una rete regolare a maglie quadrate di $500 \mathrm{~m} \mathrm{di}$ lato; per eliminare effetti anomali strettamente locali ogni stazione ì stata eseguita con quattro misure, una centrale e le altre disposte radialmente a circa $30 \mathrm{~m}$ di distanza dal centro; come valore della stazione è stato scelto il valore medio delle quattro misure.

Nell'area in studio sono state effettuate circa 11.000 stazioni. Sono stati inoltre eseguiti 9 profili est-ovest distanti l'uno dall'altro circa $10 \mathrm{~km}$, con stazioni in media ogni $5 \mathrm{~km}$, compresi fra il meridiano 
di L'Aquila e l'estremo limite orientale dell'area interessata dal vulcanismo Vicano, Sabazio e Laziale.

'Tali profili hanno lo scopo di rilevare il campo normale a varie latitudini, per la deduzione del gradiente normale di latitudine necessario alla riduzione delle misure nella zona studiata, e di acquisire la conoscenza dell'andamento delle anomalie marginali alla regione in esame, conoscenza che può contribuire alle conclusioni sulla estensione in profondità della sorgente del campo anomalo.

\section{3. - Riduzione Delle MisURE.}

Per la determinazione del campo normale in ogni stazione, indispensabile per il calcolo delle anomalie, si è proceduto per gradi.

In ogni tavoletta ̀̀ stata scelta una base, cui riferire tutte le altre stazioni; ogni base è stata a sua volta riferita ad una stazione situata al km 17 della Strada Maglianese, pochi chilometri fuori dell'area studiata, sul calcare del Lias. Poiché tuttavia nemmeno questa stazione poteva essere considerata rappresentativa del campo normale, in quanto prevedibilmente interessata dalle anomalie marginali, essa ̀̀ stata collegata con l'Osservatorio Geomagnetico di L'Aquila (Preturo), che si trova approssimativamente alla stessa latitudine, punto che è stato scelto come base di riferimento.

La riduzione per la variazione diurna, necessaria sopratutto per i nove profili fuori dell'area anomala, è stata eseguita sui magnetogrammi dell'Osservatorio di L'Aquila.

I evidente che l'anomalia di ogni singola stazione è dovuta agli effetti sia dei corpi profondi sia dei corpi superficiali, i quali ultimi non interessano nella attuale ricerca. Per ridurre gli effetti superficiali rispetto a quelli profondi si è proceduto ad un "filtraggio " dei risultati, mediando su quadrati di $2 \mathrm{~km}$ di lato, contenenti ciascuno 25 stazioni, e attribuendo il valore della media al punto centrale del quadrato; in tale modo si ritiene di aver praticamente eliminato gli effetti di uno strato superficiale dello spessore dell'ordine del centinaio di metri.

Si è ritenuto opportuno applicare la riduzione per il gradiente di latitudine non al valore delle singole stazioni originali ma ai valori "filtrati ", il che ha permesso di ottenere un notevole risparmio di lavoro, senza pregiudizio per i risultati; dallo studio dei nove profili 


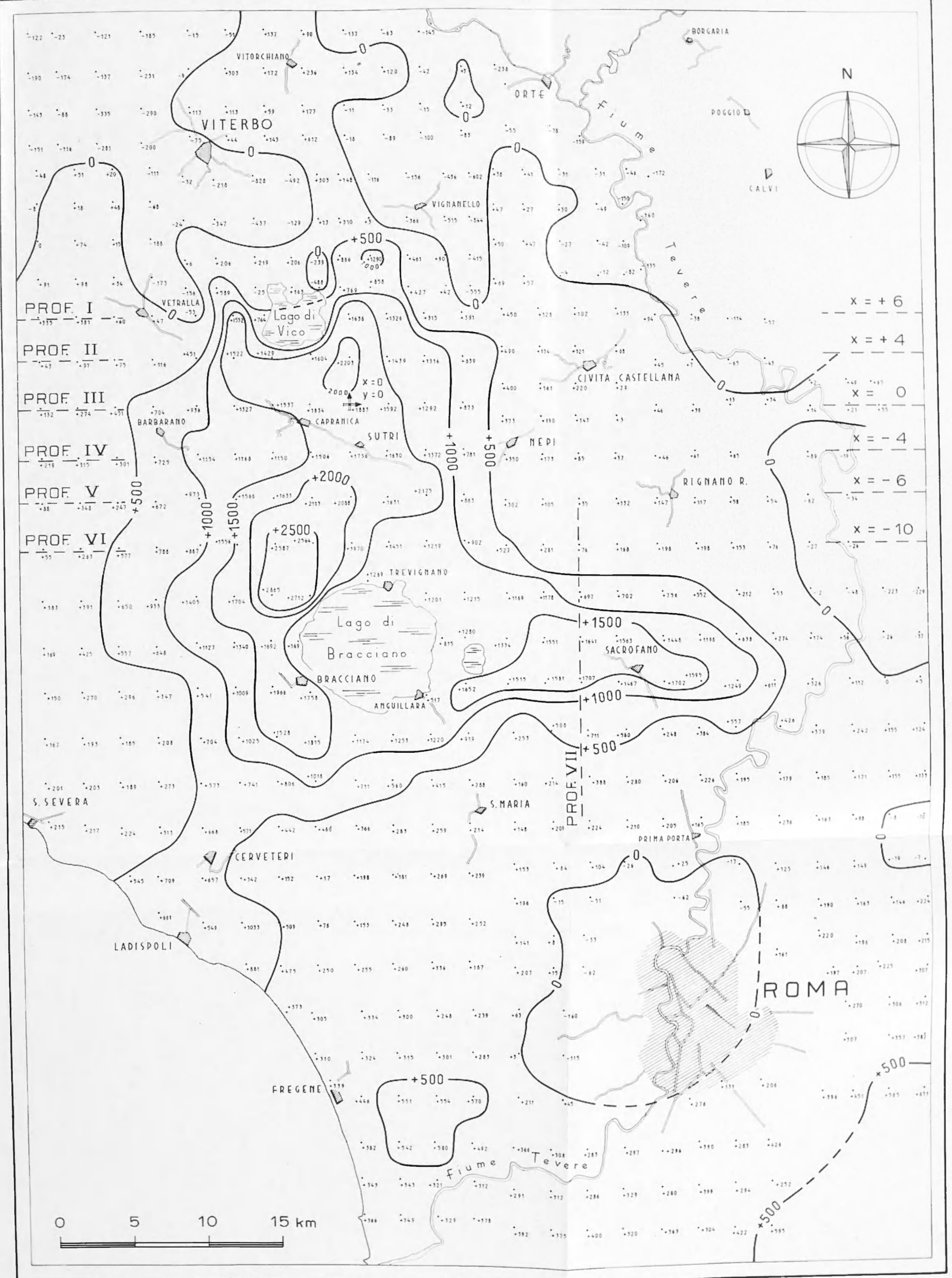




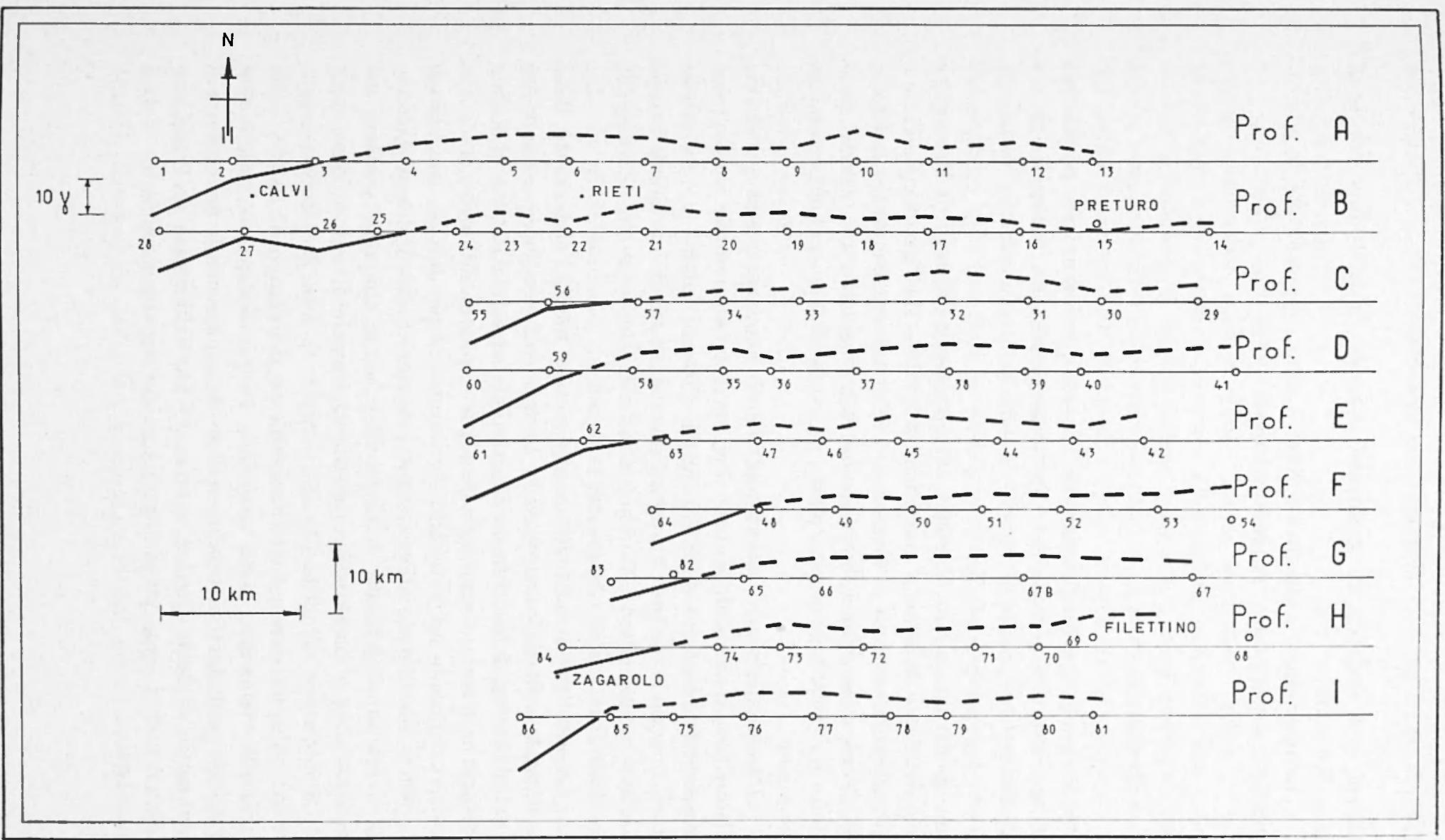

Fig. 2 - Le anomalie delle singole stazioni sono corrette per il gradiente di latitudine e riferite a L'Aquila (Preturo). Il tratto continuo nei profili indiea le anomalie negative. 
esterni si è dedotto un gradiente normale di latitudine per la $Z$ $\mathrm{di}+5,6 \gamma / \mathrm{km}$.

Le isoanomale "filtrate" e ridotte sono rappresentate in Fig. 1, mentre i profili esterni vengono riportati in Fig. 2.

\section{4. - Risultati.}

Malgrado il procedimento del filtraggio, le isoanomale presentano ancora notevoli complessità. Tuttavia si possono delineare alcune aratteristiche piuttosto chiare: la loro tendenza ad una forma ellittica leggermente allungata in direzione nord-sud e la esistenza di una apofisi laterale in direzione est all'altezza del lago di Bracciano, comprendente le conche crateriche di Polline, Martignano, Baccano e Sacrofano. Il sistema di isoanomale i inoltre caratterizzato dal fatto di essere essenzialmente positivo, raggiungendo un valore massimo di $+2856 \gamma$ in $u n$ punto a circa $5 \mathrm{~km}$ a nord-ovest di Bracciano.

L'isoanomala zero ha un andamento assai complicato; tuttavia a nord essa tende a delimitare il sistema delle anomalie centrali approssimativamente al parallelo del M. Cimino; infatti a settentrione di tale parallelo, escluso il prolungamento a nord di un debole campo positivo comprendente Viterbo, si ha un esteso campo di anomalie negrative di intensità relativamente debole.

Ad est l'isoanomala zero segue grosso modo l'andamento delle isoanomale centrali, mentre nella zona a sud essa compare soltanto a delimitare un debole campo negativo che circonda la citti di Roma. Sempre in questa zona, nella sua parte più meridionale, il campo positivo riprende ad aumentare verso sud superando la isoanomala $+500 \gamma$ con l'inizio delle prime propaggini del Vulcano Laziale. Ad ovest, sino al limite dell'area rilevata, le anomalie positive degradano sino a bassi valori senza raggiungere il valore zero.

Per quanto riguarda i profili esterni, la loro caratteristica fondamentale è un sistematico abbassamento del campo di 10-15 y nella parte più vicina alla zona vulcanica. In particolare, nei profili $C$ e $D$ che, se prolungati, attraverserebbero la zona centrale del sistema di isoanomale, i punti estremi, a circa $40 \mathrm{~km}$ di distanza dall'asse meridiano di tale zona, presentano anomalie rispettivamente di $-12 \gamma$ e $-16 \gamma$. 


\section{5. - Discussione.}

Una prima conclusione importante si può dedurre dal semplice esame qualitativo delle anomalie sopra descritte: il corpo perturbante appare chiaramente limitato alla regione vuleanica dei monti Vicani e Sabatini, il debole campo negativo a nord essendo facilmente interpretabile con il ben noto campo negativo esistente a nord del bordo settentrionale di qualsiasi corpo magnetizzato approssimativamente secondo la direzione dell'attuale campo magnetico terrestre.

ri è quindi una discontinuità fra la massa magmatica che ha alimentato il vulcanismo Ficano e Sabazio e quella responsabile del vulcanismo Fulsino. Analoga conclusione si pù̀ trarre riguardo ai rapporti con il vulcanismo Laziale a sud.

Per approfondire lo studio della regione rilevata, si è ritenuto opportuno dividere il sistema delle isoanomale in due: un sistema centrale ottenuto trascurando l'apofisi orientale e raccordando le isoanomale alla base di questa, e un sistema secondario costituito dalla suddetta apofisi.

11 sistema centrale, malgrado la complessità delle isoanomale in alcune zone particolari, presenta nel suo complesso un aspetto molto simile alle anomalie prodotte da corpi di forma geometricamente semplice come sfera o ellissoide. Per poter procedere ad una interpretazione quantitativa, si ̀̀ in un primo tempo semplificato il sistema di isoanomale, mediante l'esclusione della zona racchiusa dalla isoanomala $+2000 \gamma$, a nord-ovest del lago di Bracciano, supposta come prodotto di fenomeni puramente locali; tale semplificazione è apparsa giustificata dalla regolarità dell'andamento delle anomalie a nord di tale zona, anomalie che raggiungono nell'asse mediano del sistema un valore massimo di $+1880 \gamma$.

Il metodo di interpretazione da noi seguito è quello del confronto fra profili sperimentali, cioè dedotti dalla carta delle anomalie, in direzione est-ovest, e i corrispondenti profili calcolati per una sfera e per ellissoidi di varie dimensioni e profondità. La scelta dei profili est-ovest piuttosto che di profili meridiani è stata consigliata dalla necessità di evitare eventuali influenze degli apparati vulcanici a nord ed a sud (anche se, come si è dianzi detfo, tali influenze appaiono praticamente trascurabili), e soprattutto dalla possibilità di confrontare le anomalie negative, che si attendono da corpi di tale forma a grande distanza da essi, con quelle fornite dai profili sperimentali 
eftettuati allo scopo di determinare il campo normale (vedi n. 2 ). Tale confronto $\dot{e}$ assai utile per una valutazione della estensione in profondità del corpo allo studio, in quanto il rapporto fra i massimi valori positivi e negativi dipende appunto da essa.

Una prima approssimativa valutazione dell'entità del corpo perturbante $\dot{s}$ stata tentata considerando una sfera, tangente alla superficie, uniformemente magnetizzata in direzione verticale verso il basso.

A partire dal valore della anomalia massima $(+1880 \gamma)$ sono stati calcolati i momenti magnetici di tre sfere di raggi $5 \mathrm{~km}, 7.5 \mathrm{~km}$ e $10 \mathrm{~km}$ e da questi è stata calcolata la anomalia prodotta a $40 \mathrm{~km}$ ad est, ottenendo rispettivamente $-2 \gamma,-5 \gamma$ e $-12 \gamma$. Confrontando i valori con quelli dedotti dai profili di Fig. 2 (in particolare $i$ profili $(C$ e $D)$ si vede che la sfera di raggio $10 \mathrm{~km}$ ì quella che si adatta maggiormente ai dati sperimentali. Si è allora calcolato l'andamento delle anomalie prodotte dalla sfera lungo un profilo estovest passante sopra il suo centro; la proiezione di questo sul piano di misura è stata scelta nel punto indicato con una crocetta in Fig. 1 (immediatamente a nord di Capranica e di Sutri); tale scelta, anche se evidentemente arbitraria, appare sufficientemente giustificata dall'andamento delle isoanomale. Dato che il valore della anomalia in questo punto $\dot{a}+1880 \gamma$, per il confronto fra il profilo calcolato e quello sperimentale passante sopra il centro della sfera si è normalizzato il profilo teorico ad un valore massimo di $+1880 \gamma$. Il confronto fra i due profili è mostrato in Fig. 3 .

Il calcolo dei profili è stato eseguito anche per ellissoidi di rotazione di varie dimensioni, con il centro a profondità $h$ sotto il piano di misura, di semiasse verticale $a$ e semiassi orizzontali $b=c$. Allo scopo ì stata ntilizzata la formula del Konigsberger $\left.{ }^{1}\right)$ :

$$
\begin{aligned}
\Delta Z=3 M_{z} & \mid\left(\frac{h^{2}}{\sqrt{X}\left(a^{2}+v\right)^{3 / 2}}-\frac{l-\operatorname{arctg} l}{e^{3}}\right)+ \\
& +\frac{x h}{\operatorname{tg} I \sqrt{X}\left(b^{2}+v\right)\left(a^{2}+v\right)^{1 / 2}} \mid
\end{aligned}
$$

in cui $M_{z}$ è la componente verticale del momento magnetico del corpo;

$$
v=\frac{h^{2}+x^{2}+y^{2}-b^{2}-a^{2}}{2}+\frac{1}{2} \sqrt{X}
$$




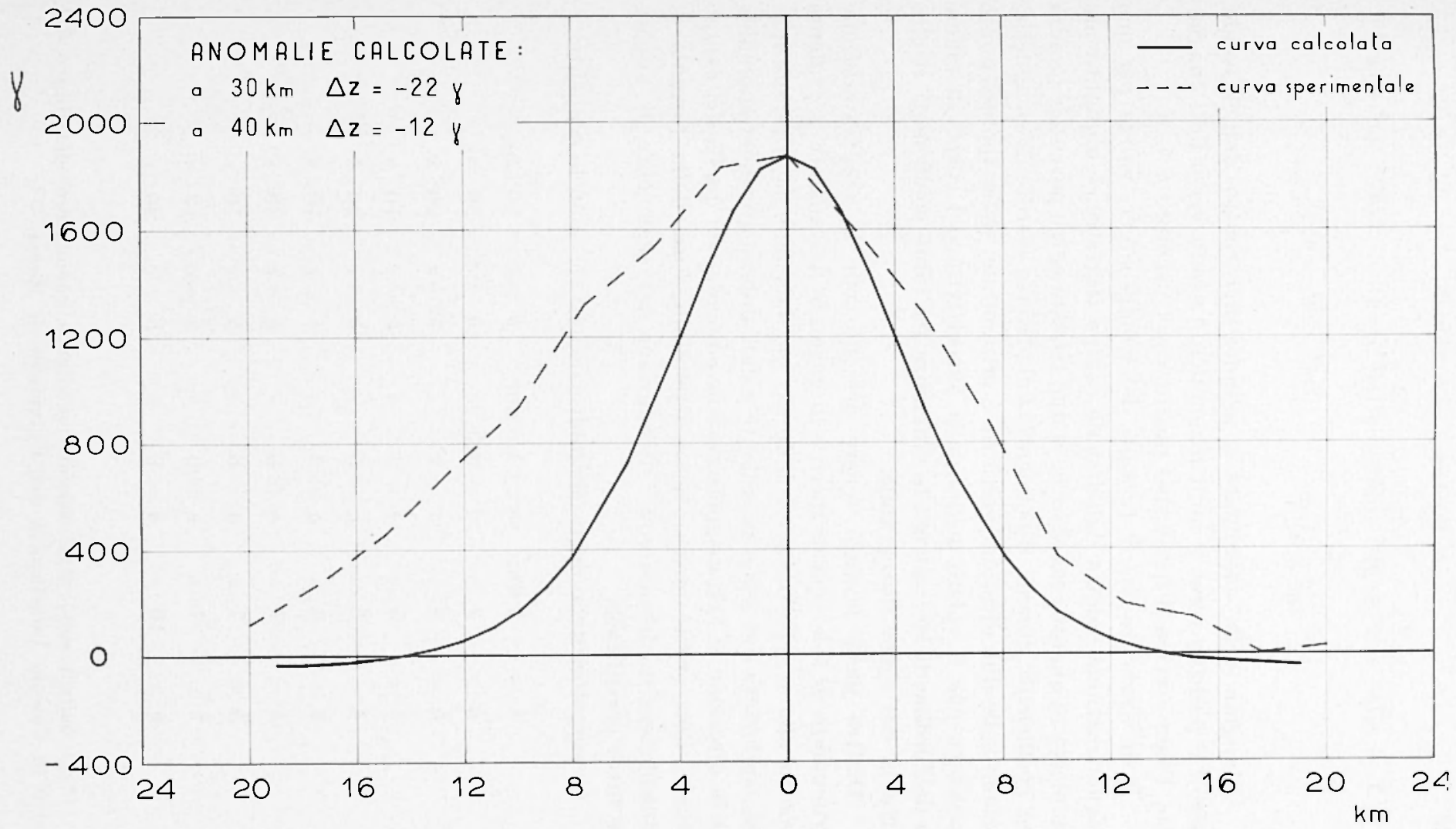

Fig. 3 


$$
\begin{gathered}
\sqrt{X}=\sqrt{\left(h^{2}+x^{2}+y^{2}\right)^{2}+\left(b^{2}-a^{2}\right)\left[\left(b^{2}-a^{2}\right)+2\left(h^{2}-\left[x^{2}+y^{2}\right]\right)\right]} \\
l=\frac{e}{\left(a^{2}+v\right)^{1 / 2}} \quad e^{2}=b^{2}-a^{2} .
\end{gathered}
$$

Jorigine delle coordinate ì assunta nel centro dell'ellissoide, lasse $x$ i positivo verso il nord magnetico, l'asse y verso l'est magnetico, l'asse $z$ verso il basso; nel nostro caso ì sempre $h<0$.

is da avvertire che la formula del Königsberger, valida per una magnetizzazione indotta dall'attuale campo terrestre, e alquanto più complessa in quanto essa associa ai due termini entro parentesi quadra due coefficienti diversi, dipendenti, dal diverso fattore di smagnetizzazione nelle due direzioni verticale e orizzontale; nella ipotesi, molto plausibile, che il valore medio della suscettivita del corpo in esame sia dell'ordine di $10^{-3}$ u.e.m., la differenza fra i due coefficienti risulta tuttavia del tutto trascurabile.

D'altra parte occorre tenere presente che la magnetizzazione permanente di tale tipo di rocce $\dot{a}$ in generale nettamente prevalente rispetto alla magnetizzazione indotta; noi facciamo la ulteriore ipotesi, confortata dal segno prevalente e dall'andamento delle anomalie, che la direzione di tale magnetizzazione coincida con quella del campo attuale. Con queste ipotesi, assai attendibili, l'uso della formula di Königsberger modificata con l'introduzione del coefficiente $\boldsymbol{M}_{\boldsymbol{z}}$ appare del tutto giustificata.

Le caratteristiche degli ellissoidi calcolati (*) sono le seguenti:

$$
\begin{aligned}
& h=-2 \mathrm{~km} \quad a=1,8 \mathrm{~km} \quad b=c=20 \mathrm{~km} \\
& h=-4 \text { " } a=3,5 \text { " } \quad b=c-20 " \\
& h=-5 " a=4 \quad \text { " } \quad b=c=20 \text { ” } \\
& h=-5 " a=4 \quad " b-r=10 " \\
& h=-6 " a=5 \quad \text { " } \quad \text { " } \quad b=c=20 \text { " } \\
& h=-6 " a=5 \quad " \quad b=c=10 " \\
& h=-7 " a=6 \quad " \quad b=c=20 " \\
& h=-7 " \quad a=6 \quad \text { " } \quad b=c=10 " \\
& h=-\tau " a=6 \quad \text { " } \quad \text { " } \quad \text { " } \\
& h=-10 " \quad a=9 \quad " \quad b=c=20 " \text {. }
\end{aligned}
$$

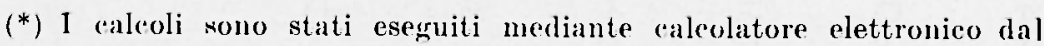
Centro di calcolo [nterfacoltà dell'Università di Roma. 

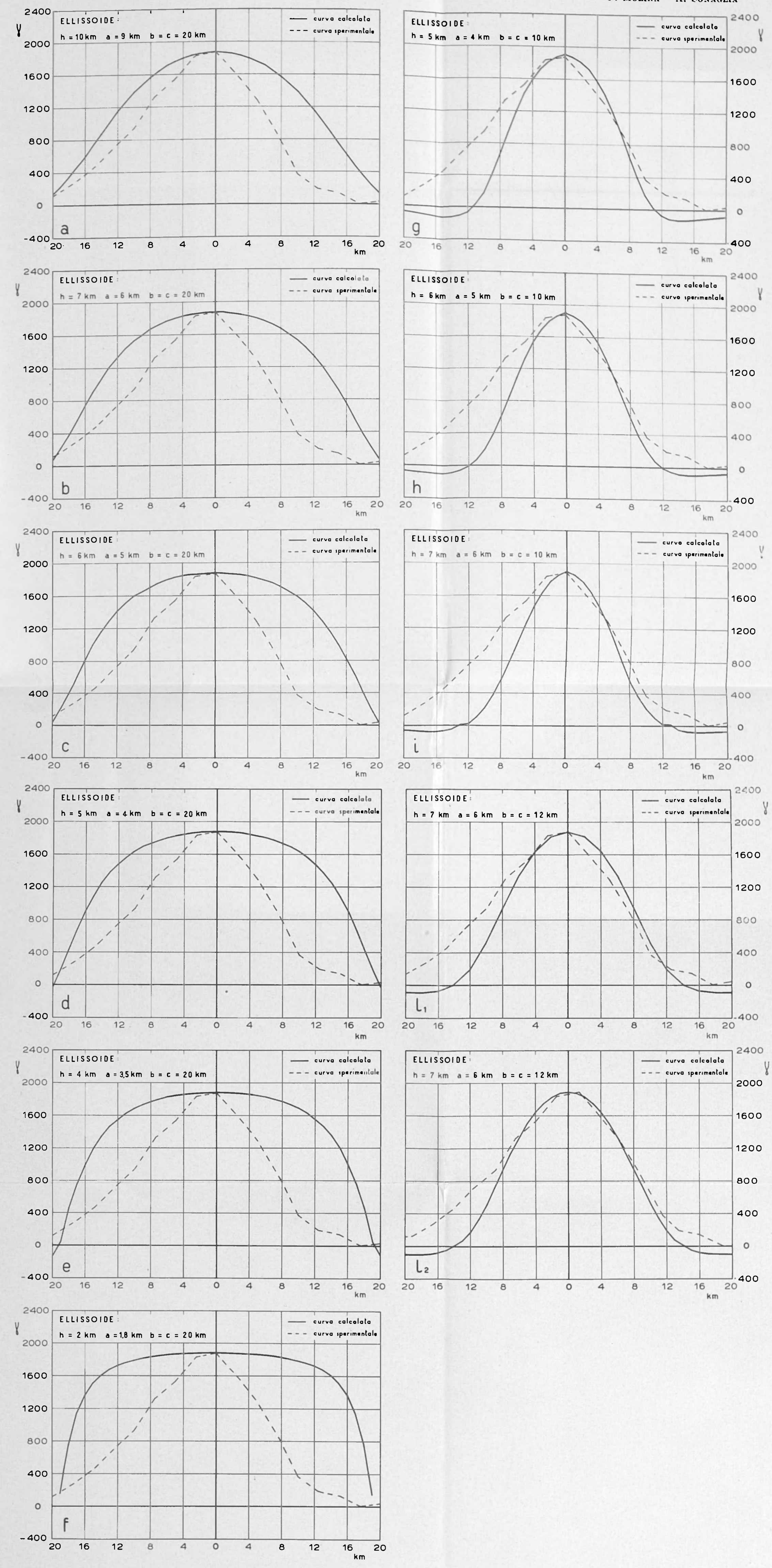
I profili est-ovest per $x=0$, normalizzati ad un valore massimo di $+1880 \gamma$, sono riportati insieme con il profilo sperimentale per il punto $x=0, y=0$, assunto coincidente col punto precedentemente scelto per la sfera, in Fig. 4. Dalla figura si nota che l'ellissoide più in accordo con i dati sperimentali è quello caratterizzato da $h=-7$ $\mathrm{km}, a=6 \mathrm{~km}$ e $b=c=12 \mathrm{~km}$ (Fig. $4 l_{1}$ ); l'accordo è ancora più evidente se si opera una traslazione di $1,25 \mathrm{~km}$ verso ovest del profilo teorico di questo ellissoide (Fig. $4 l_{2}$ ). Anche il valore della anomalia prodotta da tale ellissoide a $40 \mathrm{~km}$ ad est sul profilo centrale, cioè nel punto $x=0, y=40 \mathrm{~km},(-19 \gamma)$ è in buon accordo con il valore trovato sperimentalmente $(-12 \gamma)$ nella stazione effettuata a Montenero in Sabina (Fig. 2 - n. 55) a $41 \mathrm{~km}$ ad est del punto centrale.

Restano tuttavia delle differenze sensibili fra il profilo teoríco e quello sperimentale: quest'ultimo presenta valori alquanto più elevati a grande distanza dal punto centrale, sopratutto ad ovest, dove il gradiente è sistematicamente minore, e dove queste differenze raggiungono circa $400 \gamma$; ciò è interpretabile con una configurazione del corpo anomalo alquanto diversa da quella di un ellissoide regolare, nel senso di una maggiore estensione verso ovest rispetto a quello che è stato scelto come asse centrale. Le differenze ad est, molto minori (al massimo $200 \gamma$ ), possono invece essere interpretate come dovute alla potente coltre di tufi ivi esistente.

Malgrado le differenze ora descritte, i risultati ottenuti con i modelli di sfera e di ellissoide permettono di raggiungere una prima conclusione di massima riguardo alla forma e alla estensione in profondità del corpo perturbante, e cioè che esso può essere assimilato ad un corpo approssimativamente ellissoidale, avente il centro fra $i$ $7 \mathrm{~km}$ e $i 10 \mathrm{~km}$ di profondità e la superficie superiore molto vicina alla superficie del suolo, con dimensioni laterali dell'ordine dei $12-15 \mathrm{~km}$.

Poiché in linea di principio si sarebbe potuto ammettere anche una configurazione rappresentabile, molto schematicamente, con una coltre superficiale di materiale lavico effuso da uno stretto condotto centrale, ed il condotto stesso riempito di magma solidificato, si è ritenuto opportuno analizzare anche questa ipotesi.

Il calcolo delle anomalie prodotte da una tale configurazione è notevolmente complesso; per questa ragione è stato necessario apportare alcune semplificazioni. Si è cioè calcolato un profilo est-ovest al disopra di una fila di dipoli verticali estesa, per $20 \mathrm{~km}$, in direzione est-ovest e profonda $1 \mathrm{~km}$ (contributo della coltre superficiale); si è 


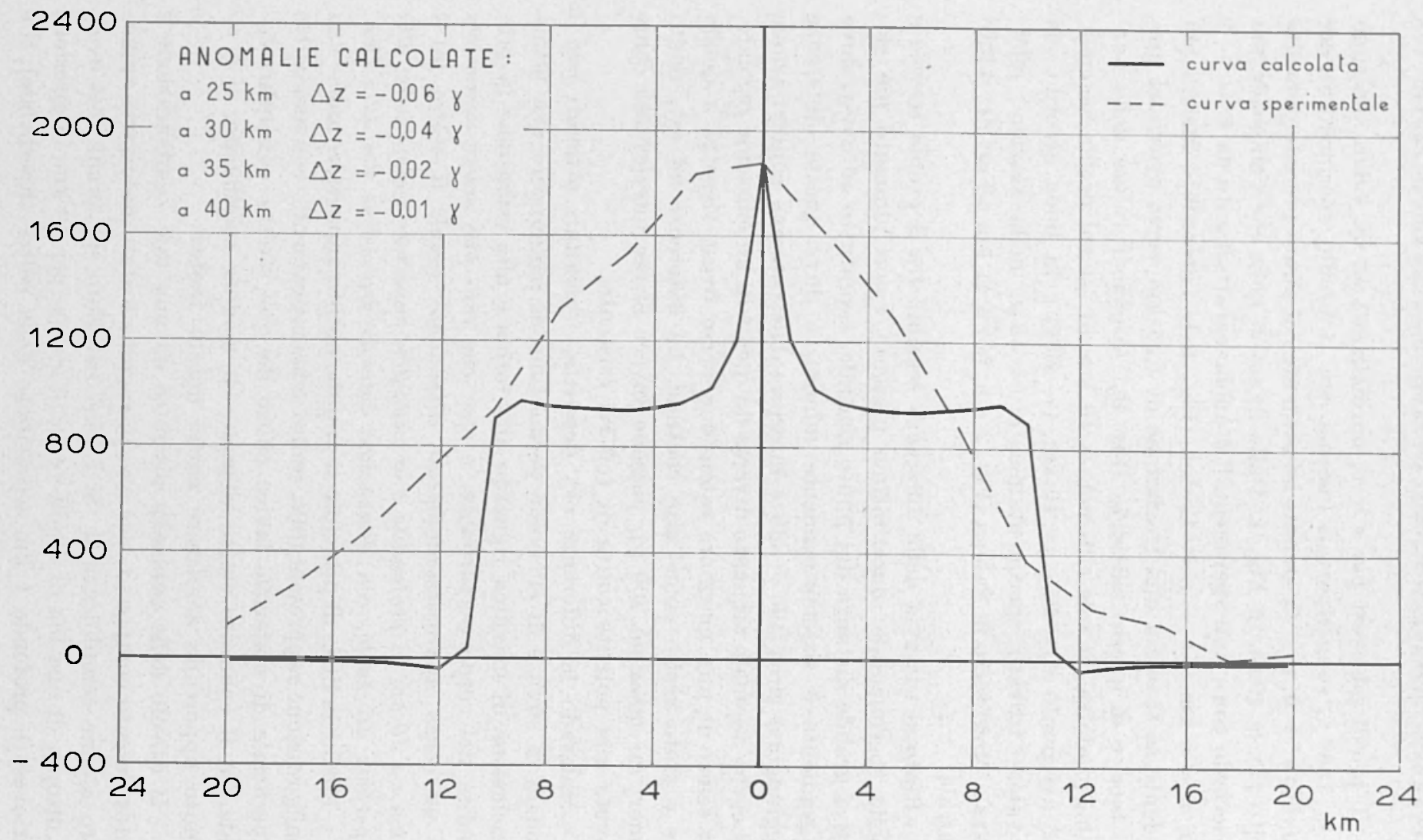

Fig. 5 
F. Molna - A. SONAGLA
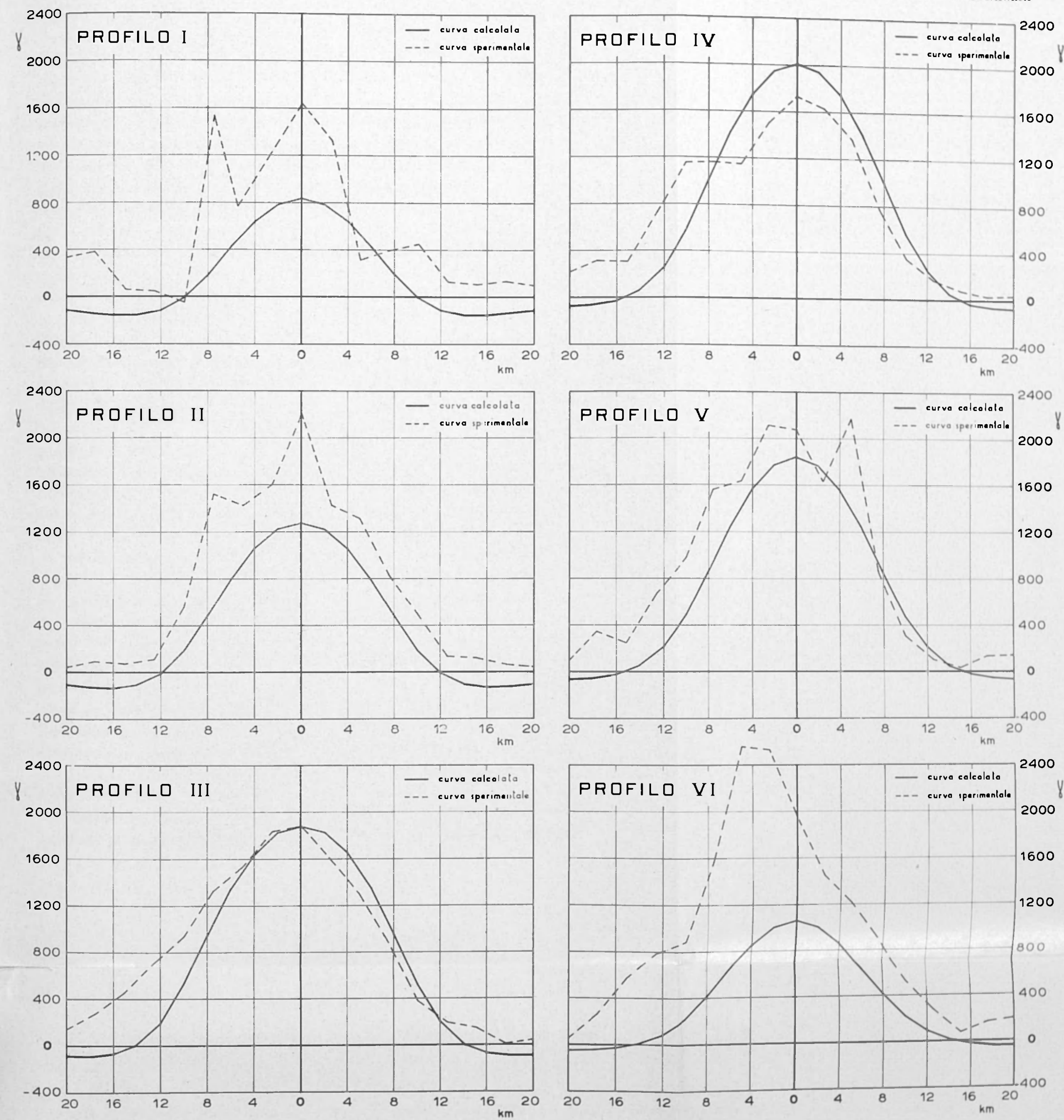

Fig. 6 
poi assimilato il condotto ad uno stretto cilindro uniformemente magnetizzato in direzione verticale verso il basso, posto al centro della coltre superficiale, lungo $10 \mathrm{~km}$ e con la superficie superiore ad $1 \mathrm{~km}$ di profondità. Il calcolo è stato effettuato applicando il metodo del Nippoldt, assimilando ciò̀ l'azione del cilindro in esame a quella di due masse magnetiche puntiformi uguali e di segno opposto, quella negativa a $1 \mathrm{~km}$ di profondità e quella positiva a $10 \mathrm{~km}$ di profondità. I risultati del confronto fra il profilo così calcolato e il profilo sperimentale (vedi Fig. 5) mostrano la nettissima differenza fra essi. Inoltre la anomalia a distanza di $40 \mathrm{~km}$, pur risultando negativa, è assolutamente trascurabile, ammontando a circa un centesimo di $\gamma$. Malgrado la estrema semplificazione del calcolo i suoi risultati non possono non rappresentare almeno le caratteristiche fondamentali delle anomalie prodotte dalla configurazione supposta; di conseguenza si ritiene che questa debba venire senz'altro scartata.

La rappresentazione del corpo perturbante come un'ellissoide, seppure valida per lo studio della estensione in profondità del corpo stesso, deve essere considerata anch'essa come una prima approssimazione. Infatti calcolando i vari profili teorici per il supposto ellissoide per vari valori di $x$ diversi da zero, risulta che l'accordo fra tali profili e quelli sperimentali viene gradualmente meno, sopratutto a nord (Fig. 6). In tale direzione le anomalie teoriche rimangono inferiori a quelle sperimentali; inoltre i profili sperimentali divengono più accidentati con gradienti assai elevati (vedi in particolare Prof. I), indicando la presenza di formazioni perturbanti superficiali; è da notare che in corrispondenza di questo profilo si è già al centro dell'apparato Vicano e deve ammettersi una risalita lungo il condotto vulcanico di questo apparato della massa magmatica, che ha complicato la forma geometrica della massa perturbante.

Verso sud l'accordo è sufficientemente buono fino ad $x=-6$ (Prof. V), anche se in questo profilo cominciano ad apparire accidentalità indicanti corpi perturbanti più superficiali; ciò d'altronde era prevedibile poiché per $x=-10$ (Prof. VI) si è già in pieno nella zona, in precedenza esclusa, a nord-ovest del lago di Bracciano, caratterizzata da anomalie di quasi $+3.000 \gamma$ e da forti gradienti. Questa zona corrisponde anch'essa ad un punto di risalita della massa magmatica, testimoniata dal campo lavico che comprende le lave leucitiche di Poggio delle Forche, M. Levo e M. Raschio.

Dalle considerazioni precedenti, nonché dall'andamento delle isoanomale possiamo ritenere giustificata l'ipotesi della esistenza di una 
massa magmatica unica di forma grossolanamente ellissoidale, avente le dimensioni precedentemente calcolate, estendentesi in direzione nord-sud all'incirca dal lago di Vico al lago di Bracciano, e che presenta delle protuberanze verso l'alto che corrispondono alla zona centrale dell'apparato vulcanico Vicano e alla zona a nord-ovest della conca del lago di Bracciano.

Anche l'apofisi orientale del sistema di isoanomale, che si estende fino alla conca craterica di Sacrofano, può essere attribuita a masse relativamente superficiali; a ciò fanno pensare i notevoli gradienti a nord e sud della apofisi stessa.

Si è tentata una valutazione quantitativa della forma e della profondità della sorgente di tali anomalie, confrontando il profilo sperimentale nord-sud (Fig. 1 - Prof. VII) con profili calcolati per varie configurazioni: cilindro-orizzontale indefinito con asse estovest $\left({ }^{2}\right)$, " vene " verticali indefinite in direzione est-ovest $\left({ }^{3.4}\right)$; ed infine linee di dipoli in direzione nord-sud, dipoli aventi una inclinazione di $58^{\circ}$.

I profili teorici che più si avvicinano a quello sperimentale sono quelli prodotti da linee di dipoli (Fig. T) i quali riproducono abbastanza bene l'andamento piatto nella parte superiore; i gradienti teorici sono più forti di quelli sperimentali, anche per la linea di dipoli più profonda $(2 \mathrm{~km})$, e ciò è probabilmente giustificato con l'osservazione che sul profilo sperimentale influiscono notevolmente, sopratutto a nord, le anomalie positive del sistema centrale.

Si può quindi ipotizzare come sorgente della apofisi orientale una specie di piastra spessa estendentesi verso est ad una profondità dell'ordine di 1-2 km, della larghezza di circa $5 \mathrm{~km}$, collegata alla massa principale.

Stabilita la forma e le dimensioni della massa magmatica perturbante si è infine tentato di valutare, sia pure approssimativamente, l'intensità di magnetizzazione media $I$ della massa stessa. Dalla normalizzazione $a+1880 \gamma$ del valore massimo del profilo centrale ottenuto dalla formula del Konigsberger da noi usata, in cui le lunghezze sono espresse in chilometri e l'intensità del campo in $\gamma$, si deduce per la costante $3 M_{z}$ il valore di $4,1 \cdot 10^{6}$; la componente verticale $M_{z}$ del momento magnetico dell'ellissoide risulta quindi $1,37 \cdot 10^{16}$ u.e.m. Dividendo per il volume dell'ellissoide $V-\frac{4}{3} \pi a b^{2}$, in cui $a=6 \mathrm{~km}=6 \cdot 10^{5} \mathrm{~cm}$ e $b=12 \mathrm{~km}=12 \cdot 10^{5} \mathrm{~cm}$, si ottiene per $I_{z}$ il valore di $3,77 \cdot 10^{-3}$ u.e.m.; supponendo come abbiamo fatto, che 


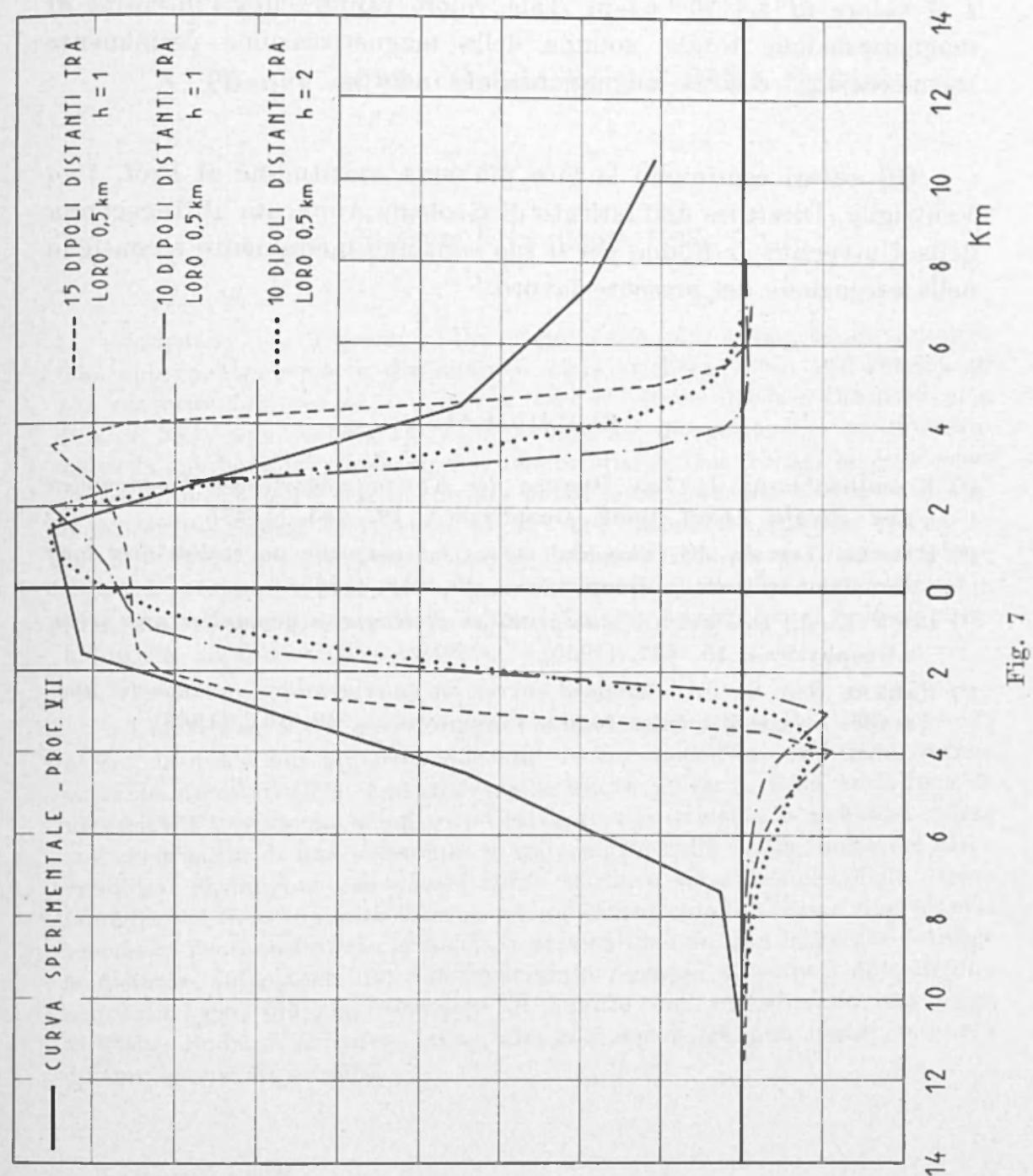

$\begin{array}{lllllllllllllll}0 & 0 & 0 & 0 & 0 & 0 & 0 & 0 & 0 & 0 & 0 & 0 & 0 \\ 0 & 0 & 0 & 0 & 0 & 0 & 0 & 0 & 0 & 0 & 1 & 1\end{array}$ 
l'inclinazione della magnetizzazione sia praticamente coincidente con l'inclinazione attuale del campo magnetico terrestre, si deduce per $I$ il valore di $4,4 \cdot 10^{-3}$ u.e.m. Tale valore rappresenta l'intensità di magnetizzazione totale, somma della magnetizzazione permanente (termoresidua) e della magnetizzazione indotta.

Gli autori esprimono la loro più viva gratitudine al Prof. Ugo Ventriglia, Direttore dell'Istituto di Geologia Applicata all'Ingegneria della Università di Roma, per il suo continuo incitamento e consiglio nella esecuzione del presente lavoro.

\section{BIBILOGRAFIA}

(1) Koenigsberger J., Zur Deutung der Karten magnetischer Isoanomalen und Profile. "Gerl. Beitr. Geophysik ", 19, 253, (1928).

(2) PakKer Gay S., Jr., Standard curves for magnetic anomalies over long horizontal cylinders. "Geophysics ", 30, 818, (1965).

( $\left.{ }^{3}\right)(00 \mathrm{~K}$ K. J., Quantitative interpretation of magnetic anomalies over veins. "Geophysies ", 15, 667, (1950).

(1) PaRker GAY S., Jr., Standard curves for interpretation of magnetic anomalies over long tabular bodies. "Geophysics ", 28, 161, (1963). 